\title{
El Uso De La Pintura Corporal En La Educación Anatómica Y Médica: Un Estudio Sustentable Interdisciplinar
}

\section{The Use of Body Painting in Anatomical and Medical Education: An Interdisciplinary Sustainability Study}

DOI: $10.46932 / \mathrm{sfjdv} 2 \mathrm{n} 2-187$

Received in: March 1st, 2021

Accepted in: May 30th, 2021

\author{
Misael Castro Serpa \\ Licenciatura en Kinesiología y Fisiatría. \\ Universidad de Montemorelos, Montemorelos, Nuevo León, México \\ Ave. Libertad 1300 pto. CP 67515 \\ E-mail: misaelcastro@um.edu.mx \\ Lilian Yolanda Rojas \\ Doctorado en Educación Familiar. \\ Universidad de Montemorelos, Montemorelos, Nuevo León, México \\ Ave. Libertad 1300 pto. CP 67515 \\ E-mail: rojasgross@um.edu.mx
}

\section{RESUMEN}

La propuesta nace del interés académico y la necesidad de analizar cómo aprenden los estudiantes de Anatomía Músculo Esquelética y qué valoran desde su percepción, al aplicar la técnica de pintura corporal anatómica como estrategia didáctica, usando materiales bajo la consigna de sustentabilidad y trabajando colaborativamente de forma interdisciplinaria. Los estudiantes guiados por docentes de Terapia Física y Rehabilitación (TFyR) y Artes Visuales (LAV), culminaron la actividad con una clase modelo. Como resultado, los estudiantes manifestaron una alta valoración ético-moral corporal, señalando la apreciación de la técnica al posibilitar su aprendizaje desde un acercamiento al cuerpo humano real en vivo. Lo que es respaldado bibliográficamente, demostrando la apreciación de la pintura corporal como complemento efectivo, económico y valorado por los estudiantes, lo que hace recomendable su implementación en el currículo de la anatomía interdisciplinar.

Palabras clave: Pintura corporal, anatomía, educación, artes visuales.

\begin{abstract}
The proposal arises from the academic interest and the need to analyze how Musculoskeletal Anatomy students learn and what they value from their perception, when applying the technique of anatomical body painting as a didactic strategy, using materials under the slogan of sustainability and working collaboratively in an interdisciplinary way. The students, guided by teachers of Physical Therapy and Rehabilitation (TFyR) and Visual Arts (LAV), culminated the activity with a model class. As a result, the students showed a high ethical-moral body valuation, pointing out the appreciation of the technique by making it possible to learn from an approach to the real live human body. This is supported bibliographically, demonstrating the appreciation of body painting as an effective, economical and valued complement by the students, which makes its implementation recommendable in the interdisciplinary anatomy curriculum.
\end{abstract}

Key words: Body painting, anatomy, education, visual arts 


\section{INTRODUCCIÓN}

Este estudio explora y evalúa el proceso de enseñanza aprendizaje de la Anatomía, mediante el uso de la pintura corporal (Finn y McLachlan, 2011; Nanjundaiah, 2012).

En este contexto ¿Qué grado de apreciación manifiestan los alumnos de Anatomía, luego de participar de una actividad que propone la pintura corporal como estrategia de aprendizaje? ¿Cuánto aprecian la retención del conocimiento anatómico y la valoración del cuerpo humano al final de la clase modelo propuesta interdisciplinariamente?

\section{ANTECEDENTES}

La investigación nace de un interés por parte de los docentes autores de la propuesta, con el deseo de facilitar el proceso de aprendizaje a través de la pintura corporal como estrategia didáctica, a fin de que los alumnos tengan la posibilidad de transferir sus conocimientos del laboratorio a un cuerpo humano vivo (Barrows, Patek y Abrahamson, 1968). Conscientes como docentes que las prácticas en laboratorio no son suficientes para evaluar la estructura u órganos de los pacientes en la práctica clínica (Pabst, 1993). Por lo que el uso de la pintura corporal en la anatomía viva ha tomado vigencia en la educación actual (Ganguly, 2010; Cookson, Aka, Finn, 2018; Jariyapong, Punsawad, Bunratsami, Kongthong, 2016).

\section{ESTADO DEL ARTE}

La pintura corporal permite la expresión artística sobre la piel humana, que puede ser un complemento útil para la enseñanza de la anatomía viva, siendo utilizado por cursos de anatomía tradicional y clases que buscan capacitar en habilidades clínicas (Finn (2010). Al implementar esta estrategia didáctica para enseñar anatomía se pintan las estructuras de músculos, vasos, huesos, nervios y órganos internos, sobre el cuerpo humano vivo y real, lo que posibilita un acercamiento al conocimiento desde un nuevo tipo de aprendizaje (Finn, 2011), en función del entendimiento anatómico (Najundaiah, 2012).

\section{OBJETIVO GENERAL}

Explorar el proceso de educación-aprendizaje de anatomía por medio de la utilización de la pintura corporal sustentable, durante una colaboración interdisciplinaria (TFyR y LAV), registrando la apreciación de parte de los alumnos (TFyR).

\section{INSTRUMENTO}

La escala de medición aplicada, explora cinco áreas en seis enunciados cuantificables con Escala de Likert 1=Ninguna, 2=Alguna, 3=Buena, 4=Muy Buena, 5=Excelente. Con una validez de constructo: 
$(\mathrm{KMO}=.574)$, una chi cuadrada de 40.792, con 10 grados de libertad, una significación de .000, y una confiabilidad alfa de Cronbach de 7.25.

Pruebas estadísticas en SPSS-22: técnica de análisis factorial, estadísticos descriptivos, de fiabilidad y prueba de efectividad.

\section{MUESTRA}

Los alumnos de primer año de las Escuelas de Terapia Física y Rehabilitación (TFyR) $(\mathrm{N}=33)$ y Artes Visuales $(\mathrm{LAV})(\mathrm{N}=4)$ de la Universidad de Montemorelos fueron invitados a participar voluntariamente de esta propuesta.

\section{MATERIALES Y APLICACIÓN}

Durante la actividad áulica se usaron pinturas corporales no tóxicas y maquillaje reciclado de modo sustentable: bases, coloretes, polvos, sombras, lápices, pinceles y aerosoles, entre otros.

Los alumnos de primer año de TFyR $(\mathrm{N}=33)$, trabajaron en cuatro grupos, con la coordinación de un alumno de LAV en cada equipo. Todos los alumnos que participaron participantes recibieron información previa sobre la actividad y no se registraron efectos adversos a la prueba de sensibilidad ante la aplicación del color. Los alumnos de LAV guiados por docentes de LAV y TFyR, llevaron a cabo la pintura corporal en los modelos. Como cierre de la actividad el maestro de TFyR dio una clase de Anatomía y mecánica del cuerpo, usando como instrumento didáctico las pinturas corporales. Posteriormente se aplicó una encuesta para explorar y evaluar el proceso de aprendizaje de los estudiantes de Anatomía.

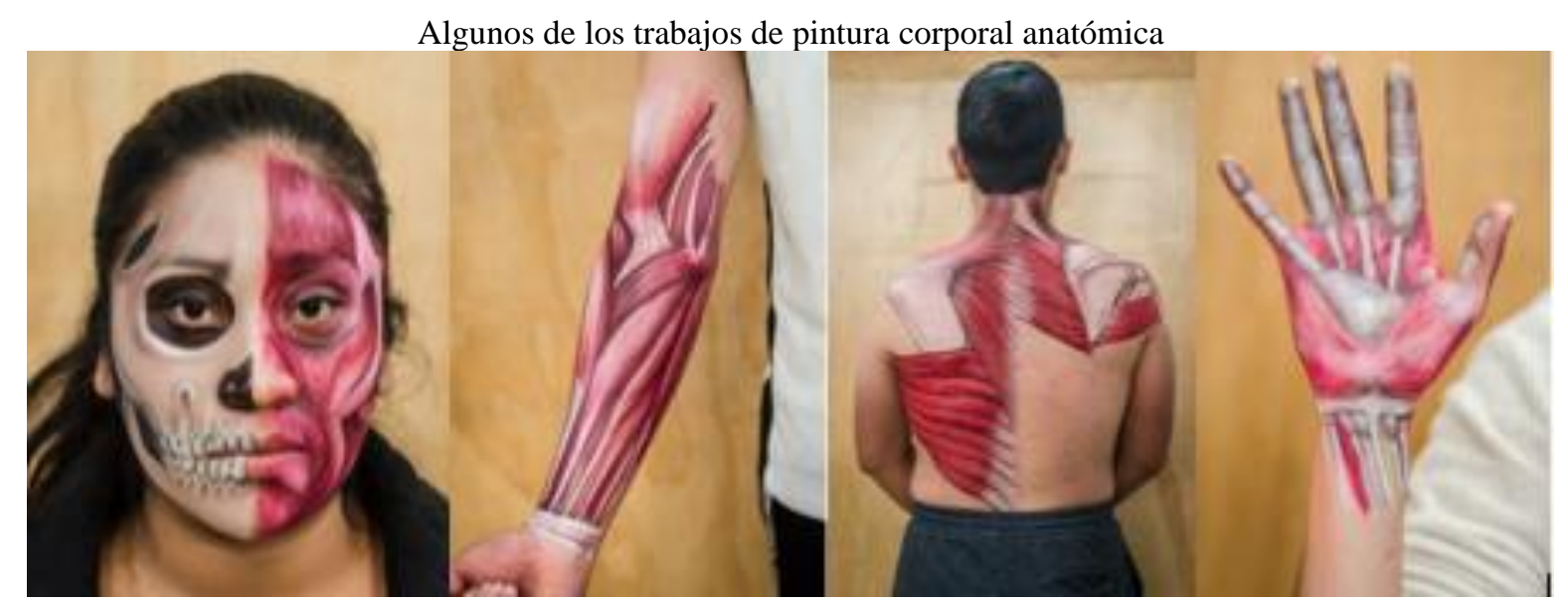

\section{RESULTADOS DE INVESTIGACIÓN}

Según la percepción de los estudiantes, los puntajes obtenidos en cada enunciado, de mayor a menor: Valoración del cuerpo humano como recurso de aprendizaje significativo Ético-Moral $(\mathrm{M}=4.88$, 
DS = .332), Eficacia de la pintura corporal como estrategia de aprendizaje de la anatomía $(\mathrm{M}=4.41$, DS $=1.121)$, Actitud frente a la pintura corporal $(\mathrm{M}=4.35, \mathrm{DS}=.931)$, Trabajo colaborativo entre pares $(\mathrm{M}$ $=4.18, \mathrm{DS}=1.074)$, Pintura corporal como estrategia de aprendizaje $(\mathrm{M}=4.12, \mathrm{DS}=1.054)$, Retención cognoscitiva $(M=3.71, \mathrm{DS}=1.047)$. Los resultados señalan que el $(97 \%)$ de los estudiantes manifiestan una apreciación ética-moral del cuerpo humano excelente. Siendo explicado el modelo con cinco factores en una varianza total de 84.971 .
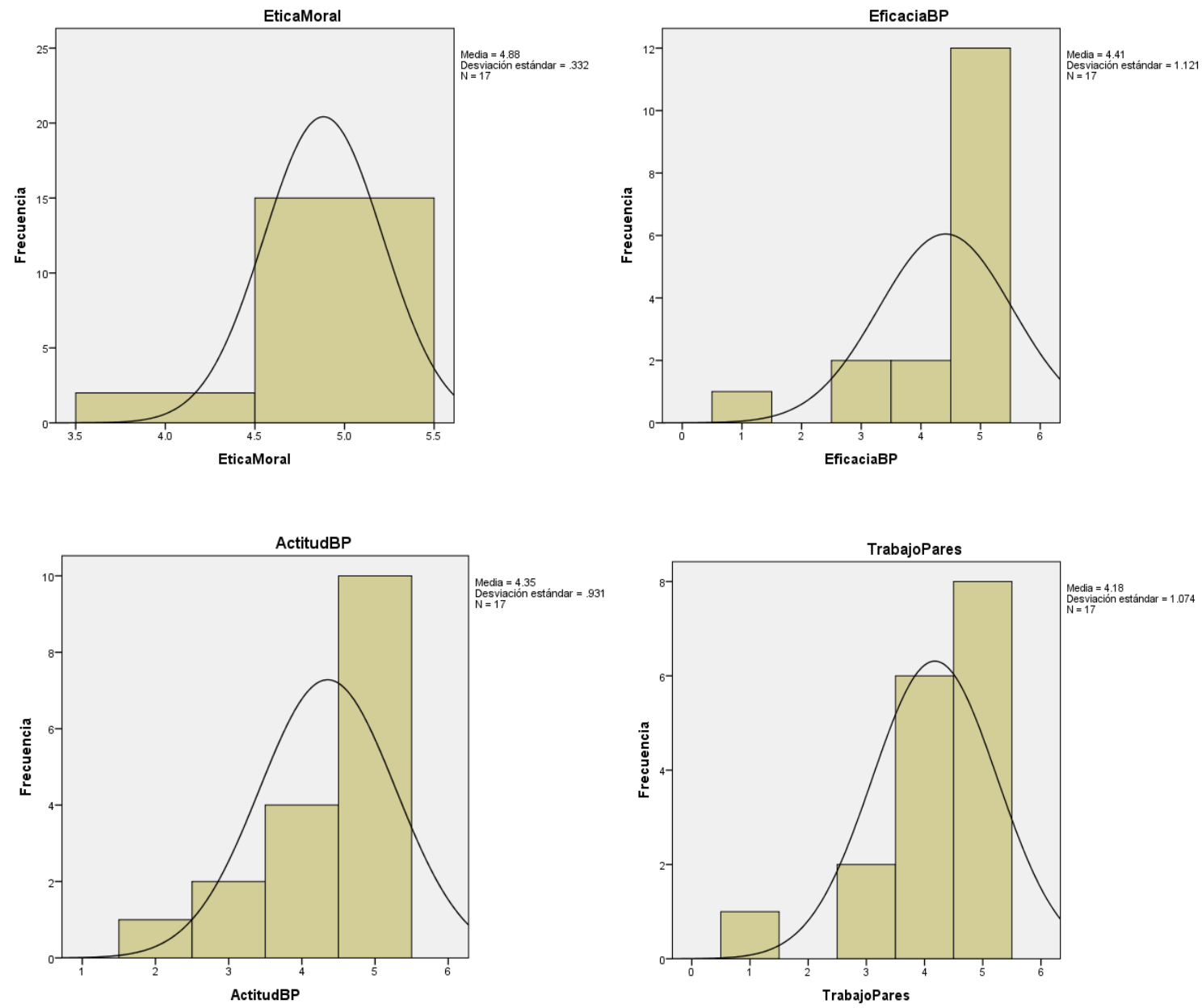

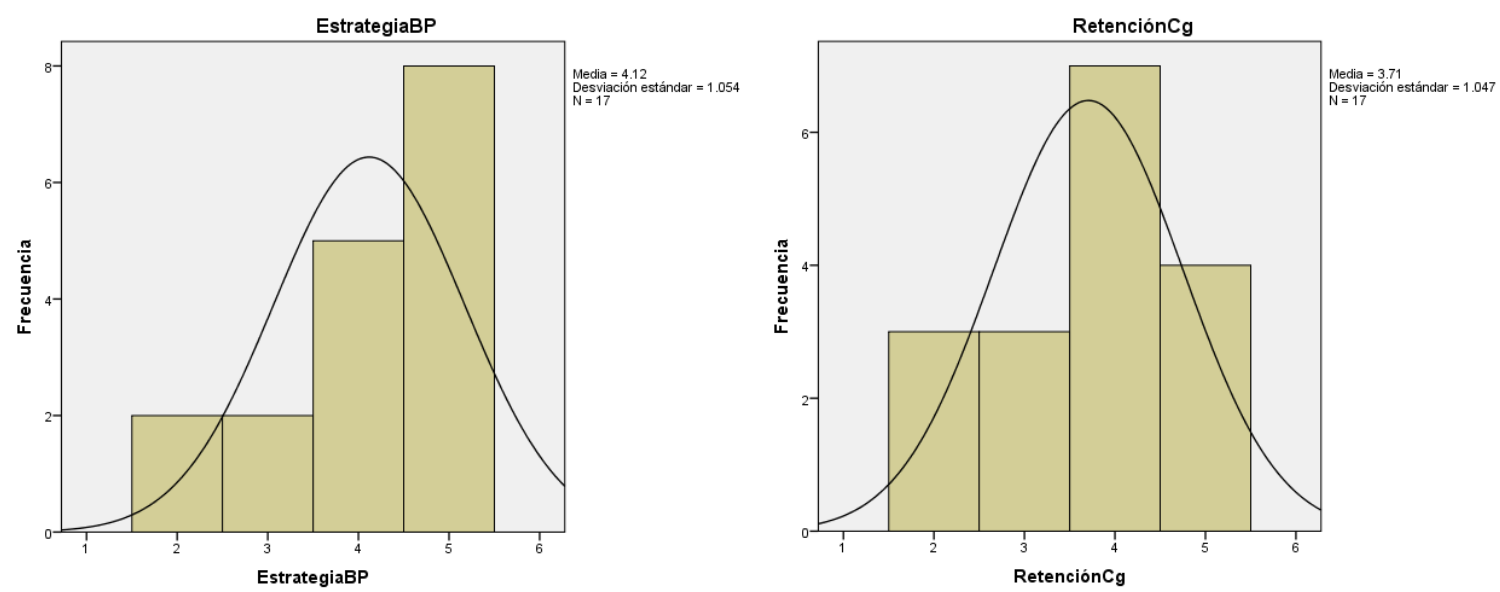

\section{CONCLUSIONES}

$\mathrm{Al}$ analizar los resultados permiten establecer a la pintura corporal como un complemento útil para la anatomía viva, cursos de anatomía clásica y clases de instrucción durante la adquisición de habilidades y competencias clínicas como lo señala (Chowdapurkar, 2011), dado que el pintar músculos, vasos, huesos, nervios y órganos internos en un cuerpo vivo, real, posibilita la simple palpación y examen anatómico.

Los resultados en general, respaldan las ventajas del aprendizaje por medio de la pintura corporal, por lo cual los autores proponen indagaciones adicionales, lo cual es consecuente con estudios anteriores que demuestran que el aprecio hacia la pintura corporal como complemento eficaz y económico al ser sustentable, es recomendable como estrategia a implementar en el currículo de anatomía interdisciplinar. 


\section{BIBLIOGRAFÍA}

Barrows, H. S., Patek, P. R., Abrahamson, S. (1968). Introduction of The Living Human Body in Freshman Gross Anatomy. Medical Education, 2(33), 5. https://www.ncbi.nlm.nih.gov/pubmed/5645770

Cookson, N. E., Aka, J. J., Finn, G. M. (2018 January 1). Exploration of Anatomists' Views toward the Use of Body Painting in Anatomical and Medical Education: An International Study. Anatomical Sciences Education [Internet], 11(2):146-54. Available from: https://search.ebscohost. com/login.aspx ?direct=true \&AuthType $=$ sso \&db=eric $\& A N=E J 1171098 \&$ lan=es \&site=ehost-live

Finn, G. M., White, P. M., Abdelbagi, I. (2011).The Impact of Color and Role on Retention of Knowledge: a Body-Painting Study Within Undergraduate Medicine. Anatomical Sciences Education, 4(6), 311-317. Retrieved from https://search.ebscohost.com/login.aspx?direct=true\&AuthType=sso\&db=eric\&AN=EJ951792\&lang=e s\&site=ehost-live8.

Finn, G.M., McLachlan, J.C. (2010). A Qualitative Study of Student Responses to Body Painting. Anatomical Sciences Education, 3(33), $8 . \quad$ Retrieved from t/publication/38094787_A_Qualitative_Study_of_Student_Responses_to_Bhttps://www.researchgate.ne t/publication/38094787_A_Qualitative_Study_of_Student_Responses_to_Body_Paintingody_Painting

Ganguly, P.K. (2010). Teaching and Learning of Anatomy in The 21st Century: Direction and The Strategies. Open Medical Education Journal, 3(5-10).

Jariyapong, P., Punsawad, C, Bunratsami, S., Kongthong, P. (2016, January). Body painting to promote self-active learning of hand anatomy for preclinical medical students. Medical Education Online, 21, 1N. http://doi.org /10.3402/meo.v21.30833.

Nanjundaiah, K. \& Chowdapurkar, S. (2012). Body-Painting: A Tool Which Can Be Used To Teach Surface Anatomy. Journal of Clinical \& Diagnostic Reseach, 6(8), 1405-1408. https://doi.org/10.7860/JCDR/2012/4700.2370

Pabst, R. (1993). Gross Anatomy: an Outdated Subject or An Essential Part of a Modern Medical Curriculum? Results of a questionnaire circulated to final-year medical students. Anatomical Tec., 237 (431), 3. https://www .ncbi.nlm.nih.gov/pubmed/8291697 